\title{
THE EFFECTS OF CONCURRENT BIOMECHANICAL BIOFEEDBACK ON NOVEL SKILL ACQUISITION
}

\section{Corresponding author:}

Anthony J. Gorman ${ }^{\mathrm{a}}$

agorman@lincoln.ac.uk

$+44(0) 1522886680$

School of Sport \& Exercise Science, College of Social Sciences, University of Lincoln, Brayford Pool, Lincoln, LN6 7TS, UK.

ORCiD: http://orcid.org/0000-0002-8886-3964

\section{Co-authors:}

Alexander P. Willmott ${ }^{\mathrm{a}}$

ORCiD: http://orcid.org/0000-0001-8895-0462

David R. Mullineaux ${ }^{\mathrm{a}}$

ORCiD: http://orcid.org/0000-0002-5688-404X

\section{Affiliations:}

${ }^{\mathrm{a}}$ School of Sport \& Exercise Science, University of Lincoln, Lincoln, UK.

Funding and grant-awarding bodies:

None

This is an Accepted Manuscript of an article published by Taylor \& Francis in Sports Biomechanics on 26th August 2019, available online:

https://www.tandfonline.com/doi/full/10.1080/14763141.2019.1650101 


\section{$1 \quad$ Abstract}

2 The aim of this study was to assess the effects of concurrent biomechanical biofeedback on the

3 ability of novices to modify relative knee, spine, and elbow motions during a rowing-type task.

4 After six non-instructed practice sessions, novices were assigned to a biofeedback $(\mathrm{BFb} ; n=$

5 7) or control group (Con; $n=7$ ), before six, ten-minute sessions of continuous rowing were

6 performed over two weeks. The $\mathrm{BFb}$ group received concurrent, visual biofeedback for

7 developing sequential timing of knee, spine, and elbow motions during the pull. Following the

8 intervention, the $\mathrm{BFb}$ group demonstrated delayed elbow flexion initiation (pre-intervention,

$946 \pm 11 \%$ pull; post-intervention, $78 \pm 3 \%$ pull; $p=0.001)$. The biofeedback further promoted

10 the consecutive ending of joint rotations (BFb: knee, $69 \pm 4 \%$ pull; spine, $73 \pm 7 \%$ pull; elbow,

$1185 \pm 3 \%$ pull; Con: knee, $79 \pm 8 \%$ pull; spine, $28 \pm 6 \%$ pull; elbow, $79 \pm 4 \%$ pull) and a move

12 towards the sequential sequencing pattern. Concurrent biomechanical biofeedback during

13 short-term training altered technique, possibly by providing guidance towards the desired

14 movement pattern and increasing error detection and correction capabilities.

15 Word Count (Abstract): 183

17 Keywords: coaching, feedback, real-time, rowing, sequencing

\section{Introduction}

Biomechanical biofeedback has been shown to be a valuable asset to motor acquisition by allowing the performer to attend to errors in the movement based on the information provided. According to the guidance hypothesis (Salmoni, Schmidt, \& Walter, 1984; Schmidt, 1991), augmented biofeedback supplements the intrinsic feedback sources of the performer, and provides increased, and potentially more specific and quantitative, information for error detection and correction to occur (Schmidt, 1991). This reduces movement error and 
accelerates adaptation as deviations from a desired pattern can be more easily and precisely detected (Winstein, Pohl, \& Lewthwaite, 1994). Despite success in a range of sports, the use of biomechanical biofeedback remains somewhat rare, possibly due to a lack of comparative studies that systematically evaluate manipulations to the biofeedback paradigm (Sigrist, Rauter, Reiner, \& Wolf, 2013). This leaves the applicability of biofeedback for optimising skill development uncertain. Both task complexity and the participant may alter the effectiveness of the type and amount of biofeedback; however, it appears that the more complex a task is the more a learner could potentially benefit from visual biofeedback (Sigrist et al., 2013), and concurrent biofeedback strategies have resulted in better task acquisition, possibly due to providing increased guidance (Park, Shea, \& Wright, 2000).

One sporting task that has been used in biofeedback studies is rowing, both ergometer and on-water. For example, consistency of work output has been modified by increasing propulsive work capacity and normalised propulsive power through providing trained rowers with visual, concurrent biofeedback on oar force and oar angle profiles on water (Spinks \& Smith, 1994). In addition, upper and lower body accelerations have been presented graphically to trained rowers, who aimed to stay within pre-determined bandwidths while ergometer rowing (Anderson, Harrison, \& Lyons, 2005). Here, both frequent, concurrent, detailed biofeedback, and summary biofeedback of the percentage of time spent outside the bandwidths, improved movement consistency (Anderson et al., 2005). Although successful, these biofeedback interventions focus on the refinement of established technique, not on the initial acquisition of a new movement pattern.

Biofeedback work with novices has assessed the attainment of movement patterns that are representative of rowing, without the desire to perform an ideal stroke. Sigrist et al. (2013) compared the effectiveness of visual, auditory, and haptic biofeedback modalities for learning a novel hand-rowing task. For three sessions, in an alternating pattern, three biofeedback 
training blocks of an average of 30 rowing cycles were conducted, each followed immediately by five cycles of no biofeedback. Visual biofeedback led to better performance in tracing a target oar path than did other modalities, and demonstrates the potential of alternating biofeedback for improving novice skill acquisition (Sigrist et al., 2013). Similarly, the present study is an assessment of the applicability of biofeedback to aid development of a rowing-type movement pattern.

The 'Rosenberg' rowing pattern is the most common rowing style, which is typified by sequential body segment movement, whereby the lower limb initiates the pull phase of the stroke, followed sequentially by trunk extension, before joint rotations of the upper limbs (Klavora, 1977; Kleshnev, 2010). While temporal sequencing is key to successful rowing, no systemic method has yet been devised for training of such timings. Development of these patterns could provide a novel complex task. In ergometer rowing, coordination between the lower limbs and the trunk segments is of importance as the trunk proposedly provides a stable lever for the transference of forces generated by the legs to the handle (Pollock, Jenkyn, Jones, Ivanova, \& Garland, 2009). At the start of the stroke, limited trunk movement is apparent over approximately the first $15-25 \%$ of the normalised length of the pull, the period associated with peak power output (Pollock et al., 2009). Similarly, rowers who initiated the pull with more extended elbows $\left(>150^{\circ}\right)$ generated $38.4 \mathrm{~N}(6.3 \%)$ greater force than those with more flexed elbows (Bompa, 1980). A reduced change in elbow flexion from the catch possibly acts as an extension of the braced position of the trunk, thus increasing the transfer of forces to the handle (Pollock et al., 2009). Between 40 and 50\% of the pull, trunk contribution to linear ergometer handle velocity equals or surpasses that of the lower limbs (Lamb, 1989). Trunk contribution peaks at approximately $70 \%$ of the pull, from which time upper and lower arm velocities provide the greatest contribution to handle velocity until the finish (Lamb, 1989). 

limbs, between approximately $0-40 \%, 40-70 \%$, and $70-100 \%$ of the pull, respectively, this temporal sequence could be used as a sequential rowing-type pattern. It is hypothesised that achieving this could be aided through the provision of concurrent biomechanical biofeedback. Hence, the aim of this study was to investigate the effectiveness of a concurrent, visual biofeedback intervention for novice acquisition of a sequential rowing-type pattern during short-term training. This information could be used to aid the development of biofeedback paradigms for incorporation into training regimes.

\section{Methods}

Participants: Fourteen females were recruited for this study (mean \pm SD; age, $20.3 \pm$ 2.1 years; height, $168.4 \pm 5.1 \mathrm{~cm}$; mass, $65.2 \pm 4.8 \mathrm{~kg}$ ). Inclusion criteria were that participants were free from injury, and had no experience of rowing or sculling, ergometer rowing, or any other rowing motion, prior to the study. Each participant gave written informed consent and visited the laboratory on twelve occasions, evenly spaced over a period of approximately four weeks. The University of Lincoln Institutional Ethical Committee granted approval for the study prior to commencement.

Data collection set-up: Passive, spherical, retro-reflective markers of $9.5 \mathrm{~mm}$ diameter were attached over the right acromion process, the lateral epicondyle of the right humerus, and the right ulnar styloid processes. Markers were also affixed over the greater trochanter and the lateral epicondyle of the right femur, and the right lateral malleolus. Further markers were placed on the spine, over the first and fifth lumbar vertebrae (L1 and L5, respectively). In

97 addition, markers were also affixed to a slide-based, Dynamic indoor rowing ergometer (Concept2 Ltd., Morrisville, VT, USA) to track handle and foot stretcher motions. The 
cord that has limited movement, as opposed to the fixed foot stretcher and free-moving seat of static ergometers. This rower was chosen as it is becoming more common for off-water training as it recreates on-water rowing more effectively than static ergometers (Elliott, Lyttle, \& Birkett, 2002) and the reduced translational movement of the participant's torso (Jongerius, Willems, \& Savelberg, 2018) increases the consistency of marking tracking. Markers were attached to the centre of the handle at its join to the pulley cable and to the top right corner of the foot stretcher. Raptor-4 Digital Cameras (Motion Analysis Corporation (MAC), Santa Rosa, CA, USA). Before each participant visit, the system was calibrated, with a maximum acceptable cut-off

110 for residuals of $0.4 \mathrm{~mm}$. The origin of the global coordinate system was orientated so that the

$111 \mathrm{X}$-axis ran horizontal and parallel to the long axis of the rowing ergometer towards the pulley

112 system, the $\mathrm{Z}$-axis was vertical and the $\mathrm{Y}$-axis was the cross product of $\mathrm{Z}$ and $\mathrm{X}$. The camera 113 and rowing ergometer set-up was positioned so that participants without altering their 114 technique could view the display of the concurrent biofeedback, which was projected onto a 115 screen in front of the participant (Figure 1).

118 Position and velocity data of the ergometer markers were taken from Cortex. All ergometer

119 linear kinematics were conducted in the sagittal (X-Z) plane, and all joint angle data were 120 calculated three-dimensionally. The elbow joint angle was defined as the angle between the vector running from the right lateral elbow marker to the right acromion marker and the vector from the right lateral elbow marker to the right lateral wrist marker (where $180^{\circ}$ was full extension). The angle between a vector running from the L5 marker to the L1 marker projected

124 onto the sagittal plane and the $\mathrm{X}$-axis was the angle of the spinal segment (where at $90^{\circ}$ the 
segment was vertical, a forward inclination of the segment towards the feet $\left(\leq 90^{\circ}\right)$ was termed

126 flexion, and a backwards inclination $\left(>90^{\circ}\right)$ was termed extension). The knee joint angle was

127 defined as the angle between the vector running from the right lateral knee marker to the marker

128 over the right greater trochanter and the vector running from the right lateral knee marker to

129 the right lateral ankle marker (where $180^{\circ}$ was full extension).

130 Two key events were defined as the instants at which the velocity of the ergometer

131 handle in the $\mathrm{X}$-axis changed from positive to negative (catch), and from negative to positive

132 (finish). These were used to define the 'pull' (catch to finish) and 'recovery' (finish to catch)

133 phases, and the combination of one pull and the following recovery constituted one rowing

134 stroke. Based on data from junior rowers rowing at 20 strokes/min, for each participant the

135 maximum stroke displacement was calculated as $83 \%$ of body height (Černe, Kamnik,

136 Vesnicer, Zganec Gros., \& Munih, 2013). When rowing, instantaneous stroke displacement

137 was calculated as the total of handle and foot stretcher marker movements from their respective

138 starting positions at the catch. This was normalised with respect to the maximum stroke

139 displacement at each time instant.

140 Biofeedback content: As identified from pilot work, novice performers tended to 141 change their elbow angle at the commencement of the pull. Consequently, to promote delayed

142 elbow flexion to develop the desired kinematic sequencing, maintenance of an elbow angle in 143 excess of $130^{\circ}$ was required over the early stages of the pull. For the content of the biofeedback,

144 the pull phase was divided into three sub-phases (I, II, and III), lasting 40,30 , and $30 \%$ of the 145 stroke displacement, respectively (Lamb, 1989). The biofeedback for each stroke was initiated 146 at the catch and was provided whilst the handle velocity was in the negative $\mathrm{X}$-axis direction

147 (i.e. towards the torso). Throughout sub-phase I of the pull, a blue dialogue box containing the 148 text 'Knee' was displayed giving instruction to produce movement through knee motion.

149 Throughout sub-phase II a lighter blue dialogue box containing the text 'Spine' gave 
instruction to use spinal motion; and throughout sub-phase III, a lighter blue dialogue box than

151 sub-phase II containing the text 'Elbow' instructed use of elbow motion. The instant the elbow

152 angle dropped to below $130^{\circ}$ during either sub-phase I or II (i.e. the first $70 \%$ of the pull), an

153 orange dialogue box appeared informing the participant that the 'elbow flexed too early'. If

154 this occurred, the orange dialogue box was displayed until the end of the current pull. The

155 biofeedback was only restarted at the next catch. Furthermore, if at any time during the pull

156 the sign of the handle marker velocity returned to being positive, or instantaneous stroke

157 displacement exceeded the normalised stroke displacement, the biofeedback switched to a red

158 box. For the duration of each recovery phase, a red dialogue box containing the text 'Recovery'

159 was displayed.

160 Data collection protocol: During each visit to the laboratory, before each test, 161 participants performed a self-selected warmup without the use of the ergometer until feeling

162 comfortable to row. During each practice and testing session, participants rowed continuously

163 for 10 minutes on the Dynamic ergometer, with damper setting of 2 and drag-factor of 100

164 (determined from pilot-testing to be comfortable for this duration), at a target heart rate between

165 130-150 beats per minute (Mackenzie, Bull, \& McGregor, 2008) provided through a FT1

166 monitor and T31 coded transmitter (Polar Electro, Kempele, Finland). If at any time heart rate

167 moved outside the range, participants were instructed to increase or decrease their rowing pace

168 accordingly. To ensure the completion of each session and to negate possible effects of fatigue,

169 heart rate was deemed a more suitable exercise target than stroke rate for novice participants,

170 given potential differences in submaximal exercise capabilities. During these sessions, no

171 information from the Performance Monitor mounted to the ergometer was visible (PM4;

172 Concept2 Ltd., Morrisville, VT, USA).

173 To give sufficient time to complete the high number of repetitions needed to replace

174 initial movement patterns with a behavioural organisation derived without augmented 

practice sessions were conducted over two weeks, during which no technique instruction or

177 biofeedback were provided during or after rowing. Kinematic data were captured for the 178 duration of the last of the preliminary visits (visit 6). Once these sessions were complete, 179 participants were randomly assigned to either a biofeedback intervention $(\mathrm{BFb})$ or a control 180 (Con) group. Participants returned to the laboratory on a further six occasions, evenly spaced over the remaining two-week period. Before the first of the intervention sessions, participants in the $\mathrm{BFb}$ group received an information sheet detailing the biofeedback protocol and the desired rowing pattern. Concurrent biofeedback was provided to the BFb group intermittently

184 for alternate minutes beginning with the second minute of each session. This was presented in 185 periods where the biofeedback appeared continuously for 1-minute, before being removed for 1-minute, equating to $50 \%$ of the total time rowing, which follows beneficial intermittent and equal-duration biofeedback paradigms for rowing-type tasks (e.g. Sigrist et al., 2013). The Con group completed the same number of remaining sessions without receiving any biofeedback.

189 Kinematic data were recorded from both groups for the duration of these sessions.

190 Data analysis: All marker identification was completed using Cortex (v4.0.1; MAC).

191 Data were exported and further analysed using custom written MATLAB code (R2015b; MathWorks, Natick, MA, USA). Coordinate data were smoothed using a zero-lag, 4th order Butterworth low-pass filter with a cut-off frequency of $7 \mathrm{~Hz}$. This was chosen after visual

194 inspection of the effects of a range of cut-off frequencies on pilot rowing data collected at the same expected stroke rate as the test data.

The last ten strokes of sessions 6, 7-11, and 12 (termed pre-intervention, sessions 1-5,

197 and post-intervention, respectively) were analysed. To assess whether the criterion for 198 successful biofeedback engagement was being met, the normalised time instant at which the 199 elbow angle decreased below $130^{\circ}$ during the pull $\left(t_{\Theta}\right)$ was calculated. In addition, to determine 
the timings of knee joint, spinal segment, and elbow joint motions, a series of breakpoints were

201 calculated. The first occurrence of an angular acceleration above $0.25 \% \mathrm{~s}^{2}\left(t_{\text {start }}\right)$ represented the start of joint or segment rotation. The first occurrence after $t_{\text {start }}$ of the angular acceleration that was more negative than $0.25 \% \mathrm{~s}^{2}\left(t_{\text {end }}\right)$ represented the initiation of slowing of joint or segment rotation. The settings for these breakpoints have been previously used for determining joint motion changes in rowing data (Gorman, Willmott, \& Mullineaux, 2016).

To account for inter-participant differences in the times the rowing stroke took to complete, all data were time normalised by cubic spline interpolation to 101 samples-per-phase of the stroke, and values were expressed as percentages of each respective phase, where $0 \%$ of the pull represented the catch, and $0 \%$ of the recovery represented the finish.

210 Statistical analysis: All statistical tests were performed in SPSS (v.21; IBM, Armonk,

211 NY, USA). Normality of data was confirmed through a non-significant Shapiro-Wilk's test ( $p>$

212 0.05). To assess the effects of the intervention on the instant the elbow angle decreased below $213130^{\circ}$, and on the start and end timings of joint and segment rotations, 2 × 2 (Group: BFb, Con;

214 Time: Pre-, Post-intervention) mixed ANOVAs were conducted. Sphericity was confirmed 215 through a non-significant Mauchly's test $(p>0.05)$. A statistical significance level of 0.05 was selected, and data were presented as means and standard deviations.

\section{Results}

There was a significant group $\mathrm{x}$ time interaction effect for the majority of the comparisons of the instant the elbow angle reduced to below $130^{\circ}\left(t_{\Theta}\right)$, and of the timing of the start $\left(t_{\text {start }}\right)$ and end $\left(t_{\text {end }}\right)$ of rotation of the knee, spine, and elbow $(p<0.05$; Table 1$)$. In general, $\mathrm{BFb}$ group timings moved towards the desired pattern whereas the Control group remained unchanged from pre- to post-intervention. Additionally, there were significant main effects of 
time for all comparisons, with post- being closer to the biofeedback pattern than preintervention $(p<0.05$; Table 1).

[Table 1 near here]

Timing of joint motions: Changes to the timing of joint motions indicated a move

towards a sequential movement pattern, such that lower limb contribution to ergometer motion ended before that of the trunk, followed sequentially by the upper limbs. The BFb group progressively delayed elbow flexion across sessions, with the desired timing of $t_{\Theta}$ being first achieved by, and maintained after, the third intervention session (Figure 2). The significant interaction (Table $1 ; p<0.001)$ indicated the $\mathrm{BFb}$ group delayed $t_{\Theta}$ from pre- $(46 \pm 11 \%$ pull $)$ to post-intervention ( $78 \pm 3 \%$ pull), whereas the Con group did not change.

[Figure 2 near here]

Before the intervention, both groups displayed elbow flexion and knee extension during sub-phase I, which persisted for most of the pull (Figure 3). However, the BFb group used knee motion during the recovery phase whereas the Con group did not (Figure 4).

[Figure 3 near here]

[Figure 4 near here]

For elbow $t_{\text {start }}$, a significant main effect of group $(F(1,13)=9.117, p=0.011)$ and significant interaction effect $(F(1,13)=14.142, p=0.003)$ was apparent. By post-intervention, $\mathrm{BFb}$ had delayed elbow $t_{\text {start }}$ until $48 \pm 27 \%$ of the pull, whereas Con only altered timing by $6 \%$. However, for knee $t_{\text {start }}$ no interaction effect was apparent $(F(1,13)=0.037, p=0.851)$, nor was there a group main effect $(F(1,13)=2.223, p=0.162)$.

There was a significant interaction for the spine segment $t_{\text {start }}(F(1,13)=7.601, p=$ 0.017), which remained consistent for $\mathrm{BFb}$ and occurred earlier in the recovery phase for Con in post-intervention (Figure 4; Table 1). Knee joint and spine segment extension therefore 
accelerated the ergometer in sub-phase I, and an absence of elbow flexion until later in the pull promoted prolonged spinal motion into sub-phases II and III.

After the biofeedback, BFb had delayed $t_{\text {end }}$ of knee, spine, and elbow motions, whereas

Con did not (Table 1; Figure 4). As such, significant interactions were apparent for $t_{\text {end }}$ of knee joint $(F(1,13)=11.259, p=0.021)$, spine segment $(F(1,13)=29.595, p=0.009)$, and elbow joint motions $(F(1,13)=8.232, p=0.014)$, alongside significant group main effects for these variables (each $p<0.05$ ), with $\mathrm{BFb}$ matching the desired sequential movement pattern better than Con (Table 1).

\section{Discussion and implications}

To assess if biofeedback is beneficial as a training aid to improve performance of complex movement patterns, concurrent biofeedback on the timing of body segment movements was provided to novices attempting a rowing-type task. Consistent with the guidance hypothesis (Salmoni et al., 1984; Schmidt, 1991) and like other novel biofeedback (Sigrist et al., 2013), the results of this study show significant guidance effects of biofeedback, as indicated by delayed elbow flexion (Figure 2). Furthermore, the participants adopted a sequential movement pattern, which was exemplified by temporal changes to the ending of knee extension (69\%), spine extension (73\%), and elbow flexion (85\% of the pull) (Table 1 ;

Figure 4). These results revealed that biofeedback was effective in guiding movement adaptation that was concomitant with the objective of the intervention, the effectiveness of which is emphasised by the relatively short period of the intervention.

The approach of this study, like that of previous feedback work (Wulf, Shea, \& Matschiner, 1998), was to provide participants with equal periods of unrestricted practice and

271 biofeedback. After practice and prior to biofeedback administration, knee extension appeared 272 to begin earlier for $\mathrm{BFb}$, and within-group differences in knee positions at the catch were 
apparent. Despite this, in both groups, all participants appeared to have systematically adopted

274 similar elbow and spine motions by the end of the pre-intervention session. This infers the use

275 of intrinsic feedback sources to provide correction to perceived errors, possibly based on the

276 common demands of the Dynamic ergometer. Such between participant similarities have

277 previously been shown during novice learning of other cyclical tasks when all feedback was withheld (Delignières et al., 1998; Nourrit et al., 2003).

Post-intervention in the $\mathrm{BFb}$ group, elbow extension was maintained until significantly

later in the pull, with the elbow angle not falling below $130^{\circ}$ later than $70 \%$ of the normalised pull (Figure 3). As such, the biofeedback promoted delayed onset of elbow flexion yet did not delay the initiation of spine extension. While participants aimed to match the sequential rowing-type pattern, specific error in the timing of joint rotation was only provided for the elbow. Although knee and spine motions affected the handle kinematics that were used to define the phases, they were not monitored directly by the biofeedback. However, within-group elbow, knee, and spine motion variability appeared reduced at the catch and throughout the

287 pull, indicating a more consistent pattern. Given the redundancy inherent in a system with multiple degrees of freedom, it is not unexpected that some aspects of this movement pattern remained unaltered. Future research should focus on how movements are adapted and controlled to produce a repeatable pattern (e.g. through dynamical systems approaches; Hamill, van Emmerick, Heiderscheit, \& Li, 1999).

The biofeedback possibly encouraged parallel processing of rhythmic, visual, and

293 kinaesthetic information that were each relevant for the generation of the intended movement pattern (Wei \& Körding, 2009). This may have promoted more specific error detection and correction to occur (Schmidt, 1991), which aided calibration of internal kinaesthetic

296 information, derived from interaction with the task, with the intended movement outcome 297 (Robin, Toussaint, Blandin, \& Proteau, 2005). Therefore, biofeedback is beneficial for 
providing detailed performance information when the accurate perception of body segment movement is potentially challenging and a kinaesthetic awareness of the correct movement

300 pattern against which to assess movement error is underdeveloped. This supports the theory 301 that biofeedback can facilitate the acquisition of motor skills and emphasises its potential as a 302 tool for the development of novel, complex skills.

303 Similar to previous studies investigating single session effects of biofeedback (Sigrist et al., 2011), participants in BFb demonstrated immediate changes to technique (Figure 2). The concurrent biofeedback therefore appeared immediately interpretable, the demands of which appeared easy to conceptualise. Thus, from the beginning of its provision, the biofeedback successfully guided participants towards the characteristics of the motion that were to be altered, possibly reducing information processing demands by mediating the general intended movement pattern (Wulf \& Shea, 2002). In addition, the rate at which the intended changes were observed and the short-term duration of the intervention reinforces the effectiveness of 311 this biofeedback. For $\mathrm{BFb}$, the influence of prior instruction cannot be ruled out. Benefits were therefore

313 from a combination of guidance from the biofeedback and the information given prior to session 1, which gave an awareness of the desired sequential rowing pattern. As such, alongside

315 the biofeedback, the instruction received could have been influential in promoting the 316 adaptation that was seen between pre-intervention and session 1 . However, the timing of elbow

317 flexion continued to be altered by $\mathrm{BFb}$ throughout the intervention, and by post-intervention,

318 elbow flexion was significantly delayed from both pre-intervention and session 1 . The 319 continued change demonstrates potentially more beneficial guidance from the biofeedback over the initial instruction and an awareness of the correct movement pattern. This provides

321 evidence that biofeedback enhanced the technique of these novice rowers beyond instruction.

322 As Con were given no information regarding the rowing pattern that was promoted by the 
biofeedback it is difficult to determine their ability to adopt the desired pattern. The impact of instruction and its potential influence on both biofeedback and control groups should be considered, particularly to clarify the effects of interventions beyond the ability of participants to self-direct change towards an instructed movement pattern.

Dynamic ergometer rowing was chosen as a model task to test biofeedback as it is complex yet relatively controlled and cyclical, and while it may not be wholly representative of either on-water or static ergometer rowing, it provided the novelty needed for these participants and lent itself to the large number of iterations needed for novices to alter their movement patterns (Nourrit et al., 2003). As there is a current paucity of research into dynamic ergometer technique, the task on which to provide biofeedback was developed using technique information derived from static ergometer and on-water rowing. Though actual rowing technique may not have been replicated, the novel and complex rowing-type movement pattern that was developed appeared suitable for testing biofeedback applicability. unfamiliar with rowing or endurance exercise would have difficulty in performing an even

338 pacing strategy. This appeared effective as during the last strokes of the last four of the intervention sessions, the $\mathrm{BFb}$ group movement patterns matched those of the biofeedback. Thus, participants were at no point fatigued to the extent that they could not perform a movement pattern that successfully corresponded to the intervention. In addition, there were no requirements to accelerate the ergometer handle or the foot stretcher as quickly as possible;

343 therefore, participants were not attempting to adjust their technique to achieve maximal 344 distance. In a similar way to increased adaptation to the rowing stroke previously being 345 observed during the recovery phase due to its greater passivity (Lay, Sparrow, Hughes, \& 346 O'Dwyer, 2002), the potentially reduced concern for force production during the pull may have 347 allowed participants to more easily change this stroke phase. However, different exercise 
intensities are used in regular rowing training (McArthur, 2002), and whilst the adaptation of

349 technique has obvious benefits for sporting applications, its transfer to conditions that more

350 closely replicate competitive performance is currently unclear.

351 This study was an investigation into the effectiveness of biofeedback as guidance for

352 novices to initially alter complex movement patterns. Post-biofeedback tests were negated as

353 the determination of any transient effects of this specific paradigm would be redundant if 354 novices could not firstly adapt to the biofeedback. This follows similar work that established 355 the usability of a paradigm for movement adaptation (e.g. Anderson et al., 2005). While clear 356 acquisition effects were apparent, the lack of retention or transfer testing means these benefits

357 are not generalisable to learning permanency as they could be transient or specific to 358 acquisition conditions due to biofeedback dependence (Park et al., 2000). Future research regarding established interventions should include assessments to detail whether movement adaptations are maintained beyond initial acquisition periods.

\section{Conclusion}

363 Guidance in the form of concurrent, intermittent, visual biomechanical biofeedback can 364 facilitate the development of novel, complex movement patterns. Successful completion of a rowing-type task was measured by determining the start and end of knee, spine, and elbow motions during the pull of the rowing stroke. Novice performers made beneficial adaptations

367 to their movement patterns, towards the sequential sequencing promoted by the biofeedback.

368 As few systemic training methods exist for the development of segmental sequencing during 369 rowing, this study shows that even relatively short-term biofeedback can provide innovative training methods that may allow technique to be changed beneficially. However, more research

371 is needed into its permanent learning effects. The integration of biofeedback into rowing 372 training programmes could be further investigated using different exercise intensities, higher 
skill levels of rower, diverse delivery paradigms, and assessments after the removal of

374 biofeedback to assess the longer-term retention of the effects.

375

376 Word Count (Main text): 4559

377

378

Acknowledgements

379

None

380

381

Disclosure statement

382

No potential conflict of interest

383

384

\section{References}

385

386

387

388

389

390

391

392

393

394

395

396

397

398

399

400

401

402

403

404

405

406

407

408

409
Anderson, R., Harrison, A., \& Lyons, G. M. (2005). Rowing: Accelerometry-based feedback - can it improve movement consistency and performance in rowing? Sports Biomechanics, 4(2), 179-195.

Bompa, T. O. (1980). Technique and muscle force. Canadian Journal of Applied Sports Science, 15(6), 245-249.

Černe, T., Kamnik, R., Vesnicer, B., Zganec Gros, J., \& Munih, M. (2013). Differences between elite, junior and non-rowers in kinematic and kinetic parameters during ergometer rowing. Human Movement Science, 32, 691-707.

Delignières, D., Nourrit, D., Sioud, R, Leroyer, P., Zattara, M., \& Micaleff, J. P. (1998). Preferred coordination modes in the first steps of the learning of a complex gymnastics skill. Human Movement Science, 17, 221-241.

Elliott, B., Lyttle, A., \& Birkett, O. (2002). The RowPerfect ergometer: A training aid for onwater single scull rowing. Sports Biomechanics, 1(2), 123-134.

Gorman, A. J., Willmott, A. P., \& Mullineaux, D. R. (2016). The determination of breakpoints in time-series data. In M. Ae, Y. Enomoto, N. Fujii, \& H. Takagi (Eds.) Proceedings of the XXXIV International Conference on Biomechanics in Sports (pp. 613-616). Tsukuba, Japan.

Hamill, J., van Emmerick, R. E. A., Heiderscheit, B. C., \& Li, L. (1999). A dynamical systems approach to lower extremity running injuries. Clinical Biomechanics, 14, 297-308. 
410 Jongerius, N., Willems, P. B. J., \& Savelberg, H. H. C. M. (2018). Different inertial properties

411 between static and dynamic rowing ergometers cause acute adaptations in coordination patterns.

412 Cogent Medicine, 5: 1478599.

413

414

415

416

417

418

419

420

421

422

423

424

425

426

427

428

429

430

431

432

433

434

435

436

437

438

439

440

441

442

443

444

445

446

447

448

Klavora, P. (1977). Three predominant styles: the Adam style; the DDR style; the Rosenberg style. Catch, 9, 13.

Kleshnev, V. (2010). Boat acceleration, temporal structure of the stroke cycle, and effectiveness in rowing. Journal of Sports Engineering and Technology, 224(1), 63-74.

Lamb, D. H. (1989). A kinematic comparison of ergometer and on-water rowing. The American Journal of Sports Medicine, 17, 367-373.

Lay, B. S., Sparrow, W. A., Hughes, K. M., \& O’Dwyer, N. J. (2002). Practice effects on coordination and control, metabolic energy expenditure, and muscle activation. Human Movement Science, 21, 807-830.

Mackenzie, H. A. M., Bull, A. M. J., \& McGregor, A. H. (2008). Changes in rowing technique over a routine one hour low intensity high volume training session. Journal of Sports Science and Medicine, 7, 486-491.

McArthur, J. (2002). High Performance Rowing (pp. 71-74). Marlborough: The Crowood Press Ltd.

Nourrit, D., Delignières, D., Caillou, N., Deschamps, T., \& Lauriot, B. (2003). On discontinuities in motor learning: A longitudinal study of complex skill acquisition on a skisimulator. Journal of Motor Behavior, 35(2), 151-170.

Park, J. H., Shea, C. H., \& Wright, D. L. (2000). Reduced - frequency concurrent and terminal feedback: A test of the guidance hypothesis. Journal of Motor Behavior, 32(3), 287-296.

Pollock, C. L., Jenkyn, T. R., Jones, I. C., Ivanova, T. D, \& Garland, S. J. (2009). Electromyography and kinematics of the trunk during rowing in elite female rowers. Medicine and Science in Sports and Exercise, 41(3), 628-636.

Robin, C., Toussaint, L., Blandin, Y., \& Proteau, L. (2005). Specificity of learning in a video - aiming task: Modifying the salience of dynamic visual clues. Journal of Motor Behavior, 37(5), 367-376.

Salmoni, A. W., Schmidt, R. A., \& Walter, C. B. (1984). Knowledge of results and motor learning: A review and critical reappraisal. Psychological Bulletin, 95(3), 355-386.

451

452

453

454

455

456

457

458

Schmidt, R. A. (1991). Frequent augmented feedback can degrade learning: Evidence and interpretations. In J. Requin, \& G. E. Stelmach (Eds.) Tutorials in Motor Science (pp. 59-75). Dordecht: Kulwer.

Sigrist, R., Schellenberg, J., Rauter, G., Broggi, S., Riener, R., \& Wolf, P. (2011). Visual and auditory augmented concurrent feedback in a complex motor task. Presence: Teleoperators and Virtual Environments, 20, 15-32. 
460 Sigrist, R., Rauter, G., Reiner, R., \& Wolf, P. (2013). Terminal feedback outperforms 461 concurrent visual, auditory, and haptic feedback in learning a complex rowing-type task. 462 Journal of Motor Behavior, 20(1), 21-53.

463

464

465

466

467

468

469

470

471

472

473

474

475

476

477

478
Spinks, W. L., \& Smith, R. M. (1994). The effects of kinetic information feedback on maximal rowing performance. Journal of Human Movement Studies, 27, 17-35.

Wei, K., \& Körding, K. (2009). Relevance of error: What drives motor adaptation? Journal of Neurophysiology, 101(2), 655-664.

Winstein, C. J., Pohl, P. S., \& Lewthwaite, R. (1994). Effects of physical guidance and knowledge of results on motor learning: Support for the guidance hypothesis. Research Quarterly for Exercise and Sport, 64(4), 316-323.

Wulf, G., Shea, C. H., \& Matschiner, S. (1998). Frequent feedback enhances complex motor skill learning. Journal of Motor Behavior, 30(2), 180-193.

Wulf, G., \& Shea, C. H. (2002). Principles derived from the study of simple skills do not generalize to complex skill learning. Psychonomic Bulletin and Review, 9(2) 185-211. 
480 Table 1 Timings of knee extension, spine extension, and elbow flexion between biofeedback $481 \quad(\mathrm{BFb} ; n=7)$ and control (Con; $n=7)$ groups (mean $\pm \mathrm{SD})$ during the rowing task.

\begin{tabular}{lcccccc}
\hline & \multicolumn{2}{c}{ Con } & \multicolumn{2}{c}{ BFb } & Int. & \\
\cline { 2 - 7 } & Pre & Post & Pre & Post & $F_{(1,13)}$ & $p$ \\
\hline Knee (\% phase) & & & & & & \\
$t_{\text {start }}$ & $3 \pm 5$ & $6 \pm 2$ & $86 \pm 16 R$ & $1 \pm 6$ & 0.037 & 0.851 \\
$t_{\text {end }}$ & $78 \pm 8$ & $79 \pm 6$ & $83 \pm 19$ & $69 \pm 4^{*}$ & $11.259^{\dagger}$ & 0.021 \\
Spine (\% phase) & & & & & & \\
$t_{\text {start }}$ & $94 \pm 12 R$ & $85 \pm 13 R^{*}$ & $83 \pm 10 R$ & $85 \pm 3 R$ & $7.601^{\dagger}$ & 0.017 \\
$t_{\text {end }}$ & $23 \pm 8$ & $28 \pm 6$ & $20 \pm 9$ & $73 \pm 7^{*}$ & $29.595^{\dagger}$ & 0.009 \\
Elbow (\% phase) & & & & & & \\
$t_{\Theta}$ & $50 \pm 11$ & $47 \pm 12$ & $46 \pm 11$ & $78 \pm 3^{*}$ & $34.119^{\dagger}$ & $<0.001$ \\
$t_{\text {start }}$ & $1 \pm 8$ & $7 \pm 8^{*}$ & $95 \pm 13 R$ & $48 \pm 27^{*}$ & $14.142^{\dagger}$ & 0.003 \\
$t_{\text {end }}$ & $77 \pm 6$ & $79 \pm 4$ & $79 \pm 6$ & $85 \pm 3^{*}$ & $8.232^{\dagger}$ & 0.014 \\
\hline
\end{tabular}

Notes: All values represent the pull phase, unless noted as the recovery $(R)$.

Int., Interaction effect; $F_{(1,13)}$, F-ratio $_{(d f)} ; t_{\text {start }}$, initiation of joint rotation; $t_{\text {end }}$, initiation of slowing of joint rotation; $t_{\Theta}$, first instance that elbow angle $<130^{\circ}$.

* Significant change between the mean of the first ten strokes of Pre- and the mean of the last ten strokes of Post-intervention $(p<0.05)$.

${ }^{\dagger}$ Significant interaction effects (group \& time) $(p<0.05)$. 


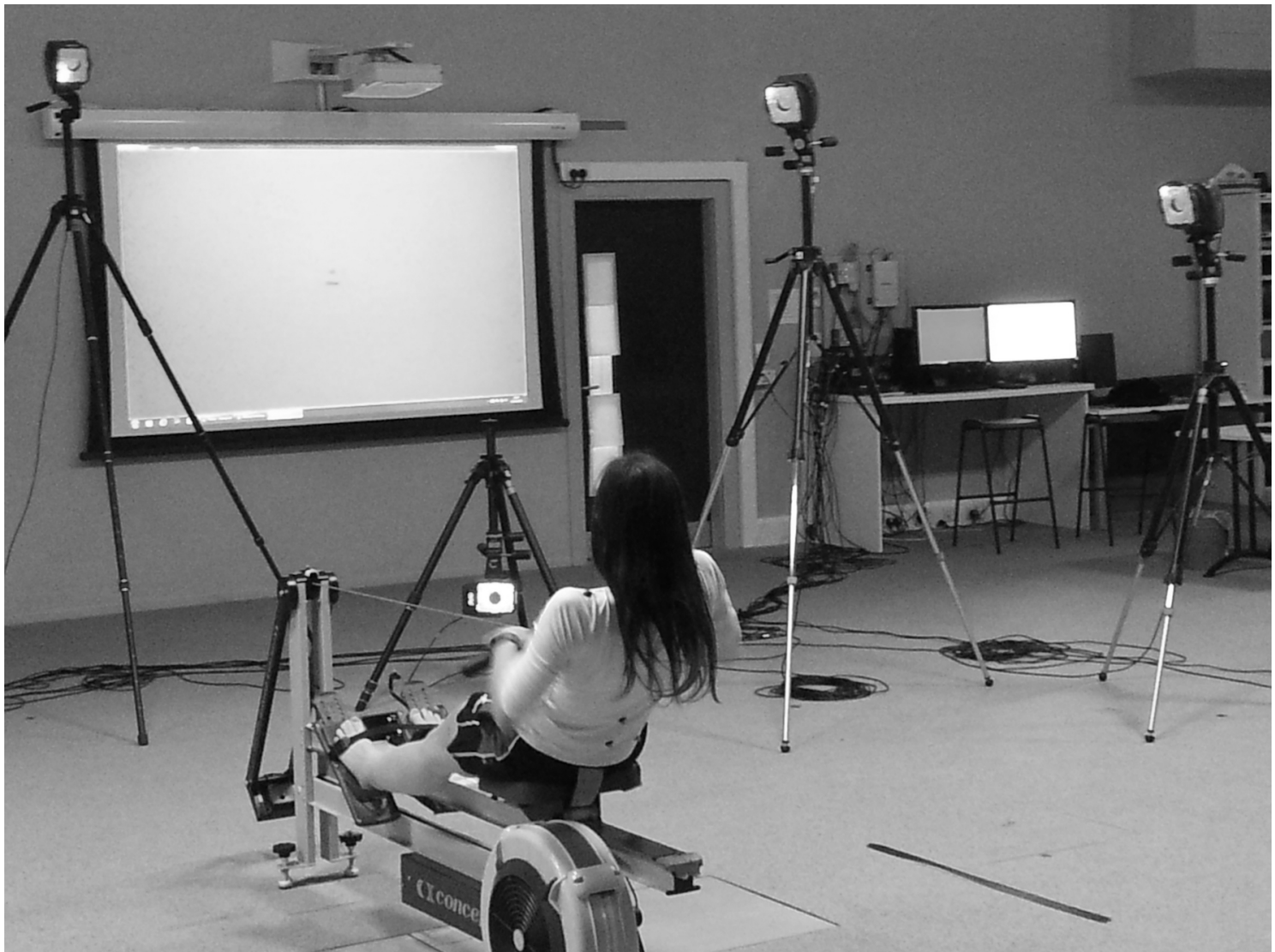

485 Figure 1 - Depiction of the data collection and biofeedback set-up showing the participant, 486 and the Dynamic ergometer position and orientation with respect to the screen where the 487 biofeedback was presented. The clothing seen in this image was not representative: during all 488 data-collection sessions, markers were attached directly to the skin, or to tight-fitting clothing. 


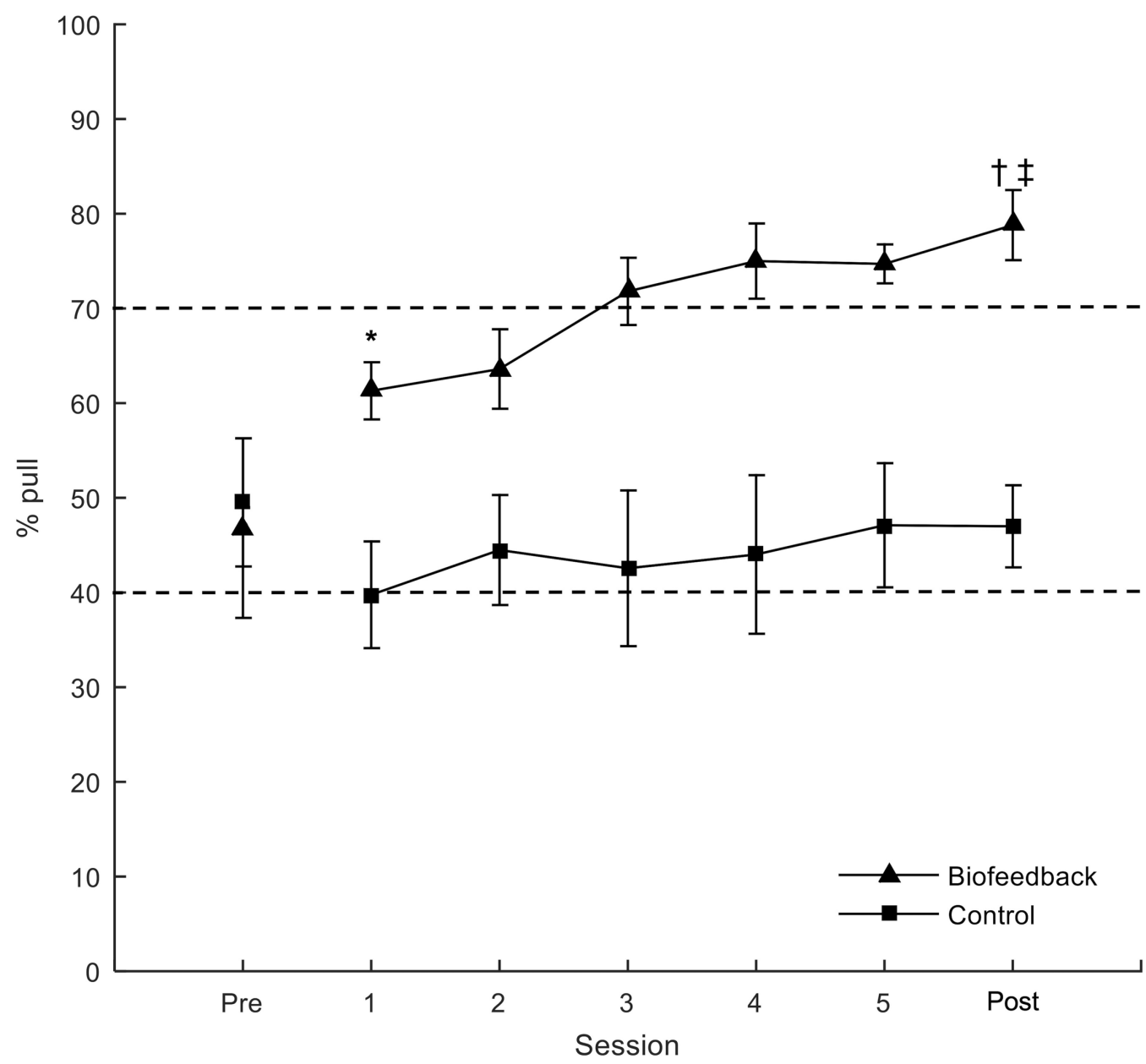

490 Figure 2 - Normalised time instants at which the elbow angle flexed to below $130^{\circ}$. Data are 491 the means of the first 10 cycles of the Pre-intervention and last 10 cycles of the five intervention 492 sessions and Post-intervention, for both the biofeedback $(n=7)$ and control $(n=7)$ groups. 493 Error bars are \pm 1 SD. Significant within group differences between Pre- intervention and 494 session $1(*)$, Pre- and Post-intervention sessions $(\dagger)$, and session 1 and Post-intervention ( $\ddagger$ ) $495(p<0.05)$. Dashed lines represent transitions between pull sub-phases $(0-40 \%$, sub-phase I; $49640-70 \%$, sub-phase II; 70 - 100\%, sub-phase III). 

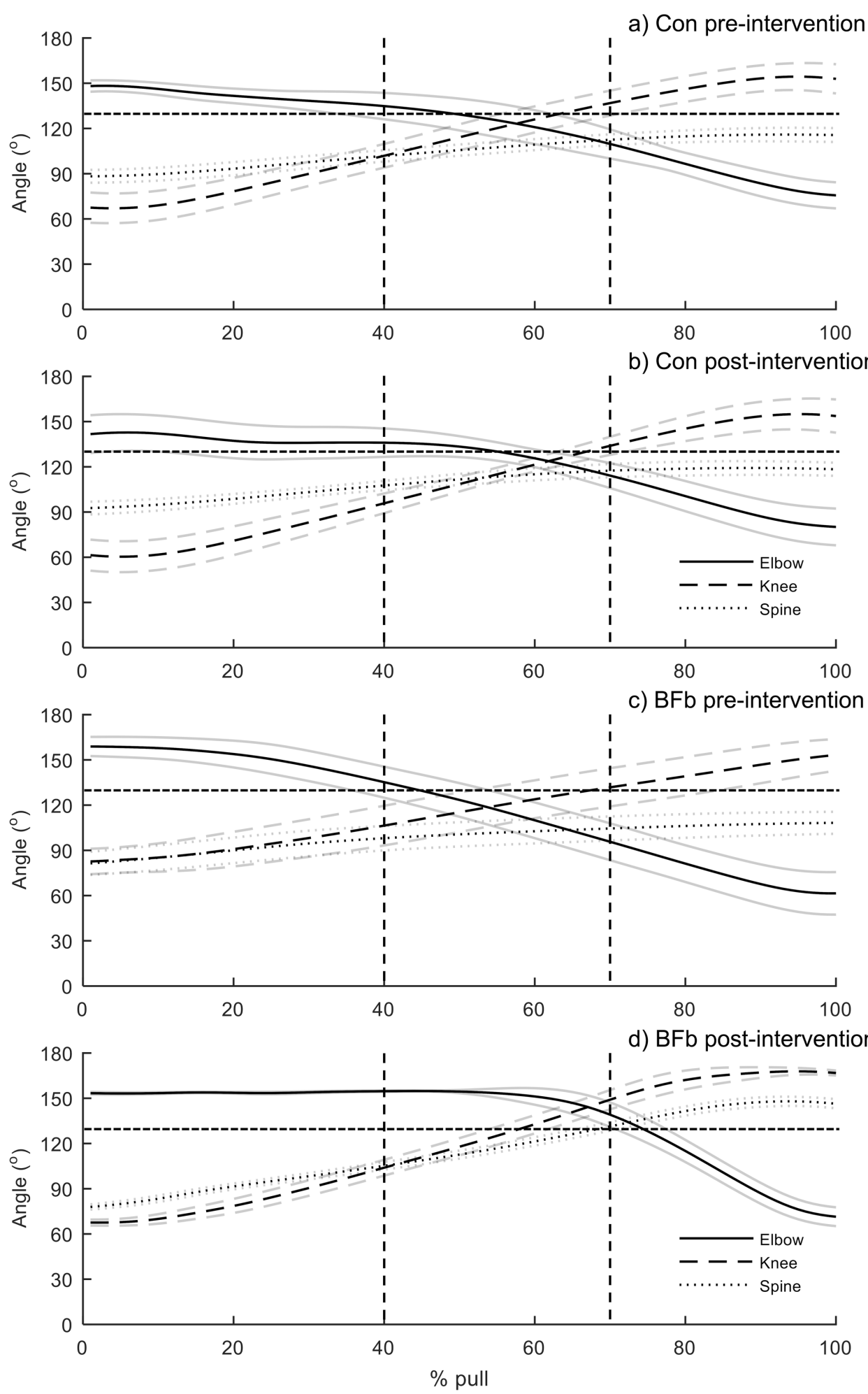

497 Figure 3 - Joint flexion-extension angle profiles during the pull for Biofeedback $(\mathrm{BFb})$ and 498 Control groups (Con), where $0^{\circ}$ is full flexion and $180^{\circ}$ is full extension. Data are means (black 499 lines) of the first ten cycles of the pre-intervention sessions and the last ten cycles of the post500 intervention session for a) Con pre-intervention; b) Con post-intervention; c) BFb pre501 intervention; and d) $\mathrm{BFb}$ post-intervention. Grey lines denote $\pm 1 \mathrm{SD}$. Vertical dashed lines 502 represent transitions between pull sub-phases (0 - 40\%, sub-phase I; 40 - 70\%, sub-phase II; $50370-100 \%$, sub-phase III), and horizontal dashed lines indicate the biofeedback targeted elbow 504 angle of $130^{\circ}$. 


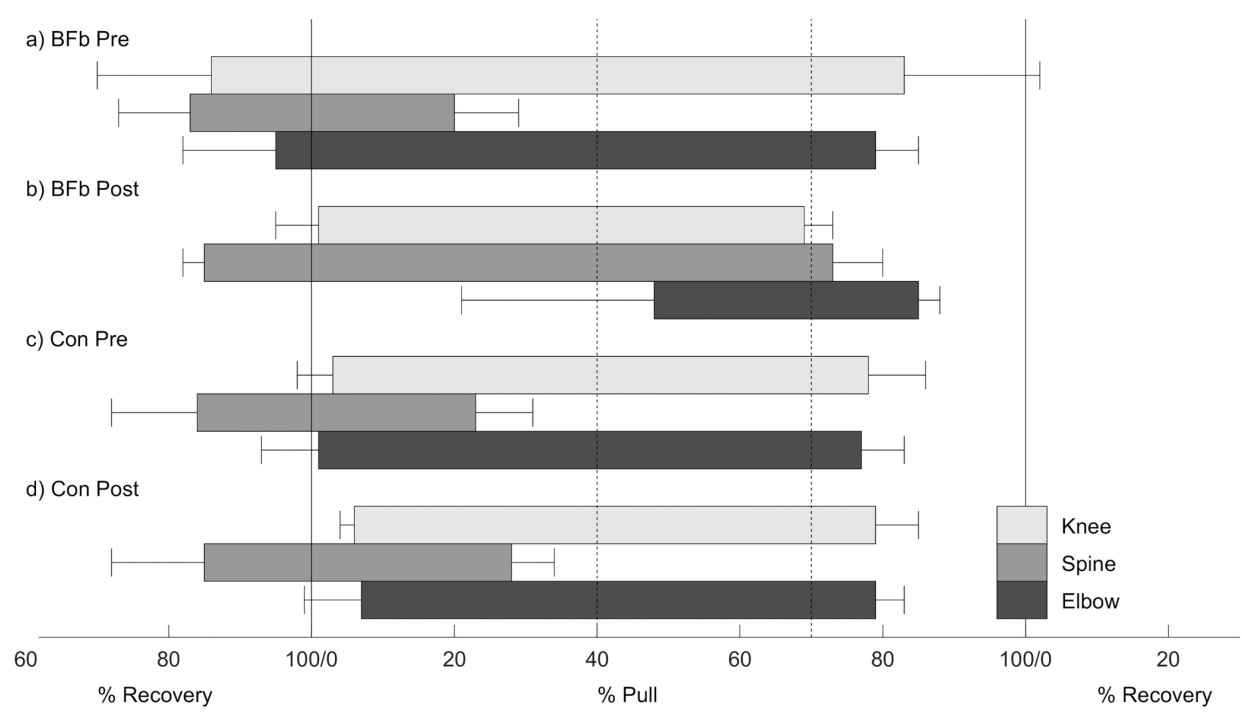

506 Figure 4 - Timing and duration of knee, spine, and elbow joint rotations in relation to the 507 phases of the rowing task for Biofeedback (BFb) and Control groups (Con). The solid 508 horizontal bars represent duration of joint rotations and error bars denote 1 SD from respective 509 joint rotation start and ends. Solid vertical lines represent transitions between pull and recovery 510 phases, while dashed vertical lines represent transitions between pull sub-phases $(0-40 \%$, sub511 phase I; 40 - 70\%, sub-phase II; 70 - 100\%, sub-phase III). Data are means of the first ten 512 cycles of the pre-intervention sessions and the last ten cycles of the post-intervention session 513 for a) $\mathrm{BFb}$ pre-intervention; b) BFb post-intervention; c) Con pre-intervention; and d) Con 514 post-intervention. 